\title{
Chitosan as an adjuvant for a Helicobacter pylori therapeutic vaccine
}

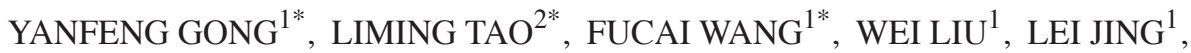 \\ DONGSHENG LIU ${ }^{1}$, SIJUN HU ${ }^{1}$, YONG XIE ${ }^{1}$ and NANJIN ZHOU ${ }^{3}$ \\ Departments of ${ }^{1}$ Gastroenterology and ${ }^{2}$ Obstetrics, The First Affiliated Hospital of Nanchang University, \\ Nanchang, Jiangxi 330000; ${ }^{3}$ Department of Biochemistry and Molecular Biology, \\ Jiangxi Medical Science Institute, Nanchang, Jiangxi 330006, P.R. China
}

Received August 28, 2014; Accepted May 13, 2015

DOI: $10.3892 / \mathrm{mmr} .2015 .3950$

\begin{abstract}
The aim of the present study was to delineate the therapeutic effect of a Helicobacter pylori vaccine with chitosan as an adjuvant, as well as to identify the potential mechanism against $H$. pylori infection when compared with an $H$. pylori vaccine, with cholera toxin (CT) as an adjuvant. Mice were first infected with $H$. pylori and, following the establishment of an effective infection model, were vaccinated using an $H$. pylori protein vaccine with chitosan as an adjuvant. Levels of $H$. pylori colonization, $H$. pylori-specific antibodies and cytokines were determined by enzyme-linked immunosorbent assay. The TLR4 and Foxp3 mRNA and protein levels were determined by reverse transcription polymerase chain reaction and immunohistochemistry, respectively. It was identified that the $H$. pylori elimination rate of the therapeutic vaccine with chitosan as an adjuvant $(58.33 \%)$ was greater than the therapeutic vaccine with CT as an adjuvant (45.45\%). The therapeutic $H$. pylori vaccine with chitosan as an adjuvant induced significantly greater antibody and cytokine levels when compared with the control groups. Notably, the IL-10 and IL-4 levels in the groups with chitosan as an adjuvant to the $H$. pylori vaccine were significantly greater than those in the groups with CT as an adjuvant. The mRNA expression levels of TLR4 and Foxp3 were significantly elevated in the mice that were vaccinated with chitosan as an adjuvant to the $H$. pylori vaccine,
\end{abstract}

Correspondence to: Dr Yong Xie, Department of Gastroenterology, The First Affiliated Hospital of Nanchang University, 17 Yongwai Zheng Street, Nanchang, Jiangxi 330000, P.R. China

E-mail: 106059546@qq.com

Dr Nanjin Zhou, Department of Biochemistry and Molecular Biology, Jiangxi Medical Science Institute, 461 Bayi Road, Nanchang, Jiangxi 330006, P.R. China

E-mail: 251315740@qq.com

${ }^{*}$ Contributed equally

Key words: chitosan, Helicobacter pylori, vaccine, adjuvant particularly in mice where the $H$. pylori infection had been eradicated. The $H$. pylori vaccine with chitosan as an adjuvant effectively increased the $H$. pylori elimination rate, the humoral immune response and the Th1/Th2 cell immune reaction; in addition, the therapeutic $H$. pylori vaccine regulated the Th1 and Th2 response. The significantly increased TLR4 expression and decreased $\mathrm{CD}^{+}{ }^{+} \mathrm{CD} 25^{+}$Foxp $3{ }^{+}$Treg cell number contributed to the immune clearance of the $H$.pylori infection. Thus, the present findings demonstrate that in mice the $H$. pylori vaccine with chitosan as an adjuvant exerts an equivalent immunotherapeutic effect on $H$. pylori infection when compared with the $H$. pylori vaccine with $\mathrm{CT}$ as an adjuvant.

\section{Introduction}

Epidemiological evidence has indicated a highly significant association between the Helicobacter pylori infection and the development of duodenal ulcers and distal gastric adenocarcinoma. In 1994, H. pylori was categorized as a class I carcinogen/definite human carcinogen by the World Health Organization (1). Current antibiotic-based therapeutic methods are not practical for global control (2), therefore, vaccines against the $H$. pylori infection are those that were developed in the past (3). H. pylori protein vaccines require an effective adjuvant (4) as $H$. pylori proteins exhibit a low immunogenicity, therefore, vaccination with an $H$. pylori antigen alone cannot induce a high enough immune response to deplete the Helicobacter infection and protect the gastric mucosa (5). Cholera toxin (CT) and heat-labile Escherichia coli enterotoxin (LT) are generally regarded as the most powerful mucosal adjuvants (6,7); however, their use in humans is hampered by their particularly high toxicities. CT and LT have been restructured to reduce their toxicities (8), however this resulted in a reduction of their adjuvant effects.

Chitosan, a polymer of D-glucosamine and a natural product derived from chitin, is accessible, and demonstrates good bioadhesion, biodegradability and biocompatibility without immunogenicity, toxicity or side-effects (9); thus, chitosan has been used in mucosal vaccines as an adjuvant (10).

Numerous studies have indicated that chitosan effectively elicits a local (particularly mucosal local) immune 
response, enhances the ability of antigenic delivery systems and performs adjuvant activity in vaccines (11). It has been reported that Neisseria meningitidis and Bordetella pertussis vaccines with chitosan as the adjuvant successfully induced a protective immune response (12).

Our previous study demonstrated that oral administration of $H$. pylori whole-cell sonicate plus chitosan as the adjuvant protected mice against $H$. pylori infection (13). Furthermore, it has been shown that, as an adjuvant in vaccines for $H$. pylori protection, chitosan is more effective than $\mathrm{CT}$ in immune protection against $H$. pylori infection (14). However, to the best of our knowledge, there have been no reports regarding chitosan as an adjuvant for the $H$. pylori therapeutic vaccine and the immunoprotection mechanism remains unclear. Therefore, in the present study, mice were infected with $H$. pylori and then vaccinated using an $H$. pylori protein vaccine with chitosan as the adjuvant. This was to delineate the therapeutic effect of the $H$.pylori vaccine and the potential mechanism against $H$. pylori infection in comparison to a $H$. pylori vaccine with $\mathrm{CT}$ as the adjuvant.

\section{Materials and methods}

Reagents and bacterial strains. Chitosan and $88.5 \%$ deacetylated chitosan powder were purchased from Shanghai Qisheng Biological Preparation Co., Ltd. (Shanghai, China). Rabbit anti-rat IgG1 (cat. no. PA1-86329; Zymed Life Technologies, Carlsbad, CA, USA), IgG2a (cat. no. 61-0220; Zymed Life Technologies) and IgA (cat. no. Sab3700520; Sigma-Aldrich, St. Louis, MO, USA), and goat anti-mouse IgG (cat. no. A27025; Zymed Life Technologies) peroxidase conjugate were purchased from Zymed Life Technologies (Carlsbad, CA, USA). CT was purchased from Sigma-Aldrich. Enzyme-linked immunosorbent assay (ELISA) kits for interleukin (IL)-2, interferons (IFNs), IL-12, IL-4, and IL-10 were purchased from eBioscience, Inc. (San Diego, CA, USA). Polymerase chain reaction (PCR) primers were purchased from Shanghai Sheng Gong Biological Engineering Technology Service Co., Ltd. (Shanghai, China) Goat anti-mouse TLR4 polyclonal antibody (cat. no. sc-12511) was purchased from Santa Cruz Biotechnology, Inc. (Dallas, TX, USA). Rabbit anti-rat Foxp3 polyclonal antibody (cat. no. bs-10211R) was purchased from Beijing Bo Orson Biological Technology Co., Ltd., (Beijing, China) and the $H$. pylori Sydney strain 1 (SS1) was provided by the H.pylori Strain Pool (Chinese Centre for Disease Control, China). An 450 enzyme microplate reader was purchased from Bio-Rad Laboratories, Inc. (Hercules, CA, USA). A PCR thermal cycler was purchased from PerkinElmer, Inc. (Waltham, MA, USA). A JS680C gel imaging analysis system was purchased from Shanghai Peiqing Science and Technology Co., Ltd (Shanghai, China) and the ECP3000 electrophoresis apparatus was purchased from Beijing Liuyi Instrument Factory (Beijing, China). A BH-2 stereo-binocular microscope was purchased from Suzhou REIT Image Technology Co., Ltd. (China).

Animals. Female BALB/c mice (age, 6-8 weeks; mean weight, $22.5 \mathrm{~g}$ ) were purchased from the Animal Center of the Chinese Academy of Sciences (Shanghai, China). The mice were housed in a specific pathogen-free environment with free access to food and water. All animal experiments were conducted in accordance with principles stated in the Guide for the Care and Use of Laboratory Animals. The experimental protocols were approved by the Ethics Committee of The First Affiliated Hospital of Nanchang University.

H. pylori culture. The H. pylori SS1 was used throughout the investigation. H. pylori was grown in a Campylobacter agar base, containing $10 \%$ sheep blood, under microaerobic conditions $\left(5 \% \mathrm{O}_{2}, 10 \% \mathrm{CO}_{2}\right.$ and $\left.85 \% \mathrm{~N}_{2}\right)$ at $37^{\circ} \mathrm{C}$ for $2-3$ days.

Preparation of H. pylori antigen. After culturing for 2-3 days, the $H$. pylori SS1 was eluted with phosphate-buffered saline (PBS) and centrifuged at $10,000 \times \mathrm{g}$ and $4^{\circ} \mathrm{C}$ for $10 \mathrm{~min}$. The pellet was washed and sonicated. Following centrifugation at $8,000 \mathrm{x} \mathrm{g}$ and $4^{\circ} \mathrm{C}$ for $30 \mathrm{~min}$, the supernatant was collected and stored at $-80^{\circ} \mathrm{C}$ until use. The protein concentration was determined using a bicinchoninic acid (BCA) assay.

Preparation of chitosan particles and solution. Deacetylated $(88.5 \%)$ chitosan powder was suspended in saline to a final concentration of $10 \mathrm{mg} / \mathrm{ml}$ and sonicated twice (output, $80 \mathrm{~Hz}$ ). The small particles in the supernatant were removed. The chitosan particles were collected by further centrifugation at $140 \mathrm{x} \mathrm{g}$ for $10 \mathrm{~min}$. Chitosan stock solution [3\% (w/w)] was prepared from $88.5 \%$ deacetylated chitosan powder in $0.8 \%$ $(\mathrm{v} / \mathrm{v})$ acetic acid and $0.9 \%(\mathrm{w} / \mathrm{v})$ saline.

H. pylori infection. Each mouse was orally administered with $1 \times 10^{9}$ colony-forming units (CFUs) of $H$. pylori per liter five times every other day. Twelve weeks after the last inoculation, four mice were euthanized, and the stomachs were removed to ascertain whether the $H$. pylori infection model had been established.

H. pylori vaccination. The infected BALB/c mice were orally immunized in the following groups at days 0, 7, 14 and 21: i) Control (PBS alone), 12 mice; ii) H. pylori antigen alone, 11 mice; iii) $H$. pylori antigen plus $0.5 \%$ chitosan solution, 12 mice; iv) $H$. pylori antigen plus CT (5 $\mu \mathrm{g} /$ mouse), 11 mice (one mouse died); and v) H. pylori antigen plus chitosan particles (500 $\mu \mathrm{g} /$ mouse), 12 mice. Four weeks after the final vaccination, saliva and blood samples were collected. The stomachs were isolated and cut longitudinally into two sections. One was used for examination of $H$. pylori, and the other was used for histology and immunological assays.

Assessment of bacterial load in the stomach. The bacterial load in the stomach was determined by quantitative culture of $H$. pylori and Giemsa staining. H. pylori-negative was defined when the culture of $H$.pylori and the Giemsa staining were negative. $H$. pylori-positive was defined when either the culture of $H$. pylori or the Giemsa staining was positive. For assessment of $H$. pylori colonization, the weighed stomachs were homogenized in Brucella broth and spread over a serum plate. The plates were incubated for 3-7 days, and the H.pylori colonies were counted and calculated as CFUs per gram of stomach tissue. For Giemsa staining, the colonization was assessed by semi-quantitative analysis of $H$. pylori in the 

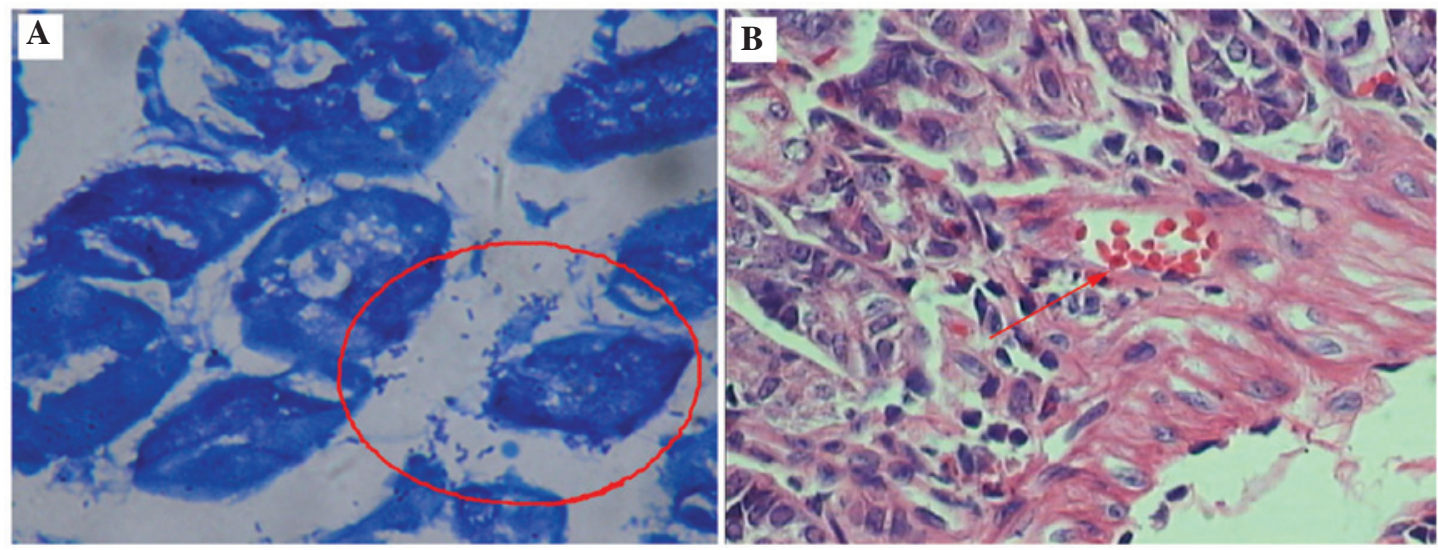

Figure 1. (A) Giemsa staining of the gastric mucosa (magnification, $\mathrm{x} 400$ ). Mice were euthanized 12 weeks after the $H$. pylori inoculation and the tissue sections were stained by Giemsa staining. The red circle indicates H. pylori colonization in the gastric pits. (B) Hematoxylin and eosin staining of the gastric mucosa (magnification, x400). Mice were euthanized 12 weeks after the $H$. pylori inoculation. The red arrow indicates mucosal hyperemia, lymphocytes and neutrophil infiltration.

gastric mucosa ( 0 , nil; 1, 1-2 cells/crypt; 2, 3-10 cells/crypt; 3 , 11-20 cells/crypt; and 4, >21 cells/crypt).

Determination of $H$. pylori-specific antibody levels in the gastric mucosa and saliva. The $H$. pylori-specific antibodies, IgG, IgG1, and IgG2a in sera, and IgA in the gastric mucosa and saliva were detected by indirect ELISA. After weighing, the gastric mucosa was homogenized in PBS and the homogenates were centrifuged at $3,000 \times \mathrm{g}$ at $4^{\circ} \mathrm{C}$ for $20 \mathrm{~min}$. The supernatant was harvested and diluted at 1:2. The sera and saliva were diluted at $1: 100$ and $1: 5$, respectively. Peroxidase-conjugated rabbit anti-rat IgG1, IgG2a or IgA was diluted at 1:1,000 and peroxidase-conjugated goat anti-mouse IgG secondary antibody was diluted at 1:2,000. The antibody levels of each immunized group from the sera and saliva were represented as relative levels to the mock-immunized control group. The IgA levels in the gastric mucosa were represented as relative levels (per gram wet weight of the gastric mucosa) to the mock-immunized group.

Determination of cytokines in the gastric mucosa by ELISA. After weighing, the gastric mucosa was homogenized in PBS and the homogenates were centrifuged at $3,000 \times \mathrm{g}$ at $4^{\circ} \mathrm{C}$ for $20 \mathrm{~min}$. ELISA kits were used to quantify IL-2, IFN, IL-12, IL-4 and IL-10 in the supernatants (diluted at 1:2) following centrifugation. The results were represented as $\mathrm{pg} / \mathrm{mg}$ wet weight of the gastric mucosa.

Determination of TLR4 and Foxp3 mRNA contents in the gastric mucosa by reverse transcription (RT)-PCR. Total RNA was isolated from the mouse gastric mucosa to determine TLR4 and Foxp3 mRNA levels within the gastric mucosa using RT-PCR. The cDNA from each sample served as a template for subsequent PCR assays to assess the TLR4 and Foxp3 mRNA levels, which were normalized to the expression of $\beta$-actin. Each 50- $\mu$ l PCR consisted of 25 pmol of each primer, $10 \mathrm{mM}$ Tris ( $\mathrm{pH} 8.3$ ), $1.5 \mathrm{mM} \mathrm{MgCl}_{2}, 200 \mu \mathrm{M}$ dNTPs, and $0.5 \mu \mathrm{l}$ of Taq enzyme. $\beta$-actin and TLR4 were amplified at $95^{\circ} \mathrm{C}$ for $5 \mathrm{~min}(1 \mathrm{cycle}) ; 95^{\circ} \mathrm{C}$ for $30 \mathrm{sec}, 56^{\circ} \mathrm{C}$ for $30 \mathrm{sec}$ and $72^{\circ} \mathrm{C}$ for $1 \mathrm{~min}\left(30\right.$ cycles); and $72^{\circ} \mathrm{C}$ for $5 \mathrm{~min}$ ( 1 cycle).
Foxp3 was amplified at $95^{\circ} \mathrm{C}$ for $2.5 \mathrm{~min}(1$ cycle $) ; 95^{\circ} \mathrm{C}$ for $30 \mathrm{sec}, 57^{\circ} \mathrm{C}$ for $30 \mathrm{sec}$ and $72^{\circ} \mathrm{C}$ for $1 \mathrm{~min}(32$ cycles $) ; 72^{\circ} \mathrm{C}$ for 5 min ( 1 cycle). The PCR products were visualized following electrophoresis on $2 \%$ agarose gels and the band intensities were quantified by densitometry.

Determination of TLR4 and Foxp3 protein expression in the gastric mucosa by immunohistochemistry. Snap-frozen biopsies were cut into $4-\mu \mathrm{m}$ sections to determine the levels of TLR4 and Foxp3 protein expression in the gastric mucosa by immunohistochemistry. In the TLR4-stained sections, positive cells were assigned to the cell membrane or to yellow/brown-dyed plasma, and negative cells were assigned to the cell membrane or to non-dyed plasma. In Foxp3-stained sections, positive cells were assigned to the cell nucleus or to yellow/brown-dyed plasma and negative cells were assigned to the cell membrane or to non-dyed plasma. The immunoreaction was graded according to the depth of the color and the proportion of positive cells. The degree of dyeing was divided into the following score grades and expressed as a percentage: 0 , Negative; 1, (yellow) weakly positive; 2 (light brown) positive; and 3 (brown) strongly positive.

Statistical analysis. Differences in the eradication rate were analyzed by Fisher's exact test. Differences in H.pylori-specific antibody levels in the gastric mucosa among the experimental groups were evaluated for statistical significance by analysis of variance or Student's t-test. $\mathrm{P}<0.05$ was considered to indicate a statistically significant difference.

\section{Results}

Animal model of $H$. pylori infection. The animal model of $H$. pylori infection was established for the different infection groups. Four mice were euthanized 12 weeks after oral infection with $H$. pylori. Positive $H$. pylori-infection was observed by $H$. pylori culture and Giemsa staining of sections of the infected stomachs (Fig. 1A). Gastric inflammation was observed by hematoxylin and eosin (H\&E) staining (Fig. 1B), thus indicating the H.pylori infection. 
A

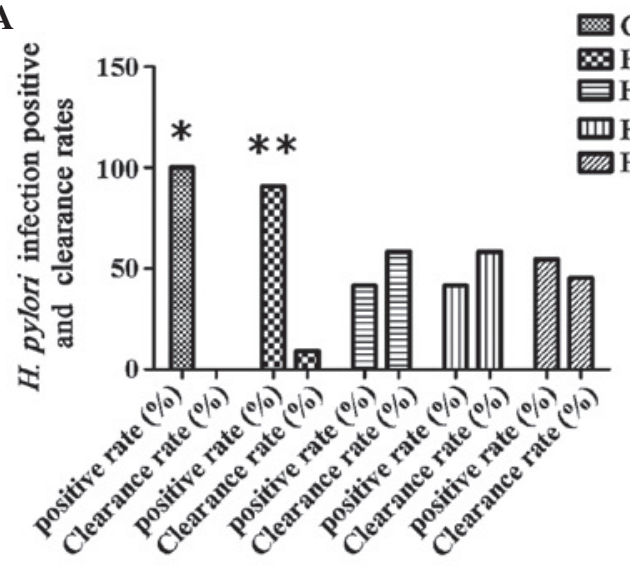

C

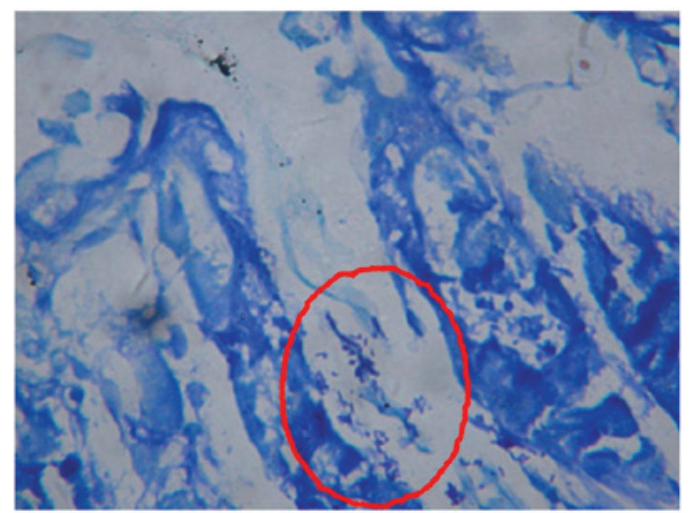

D

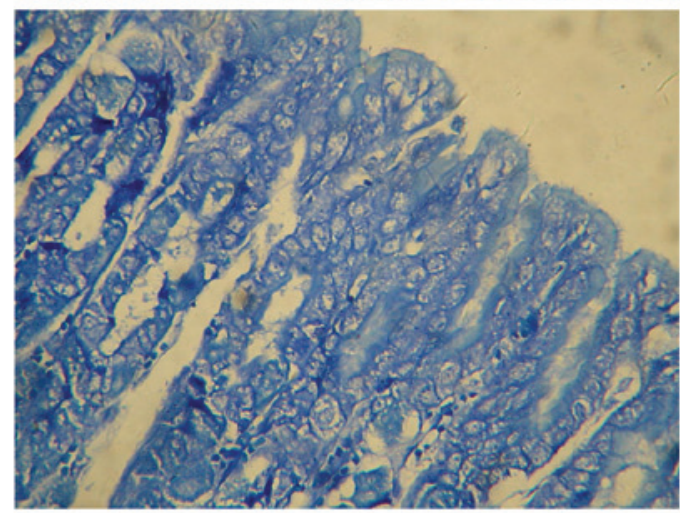

B

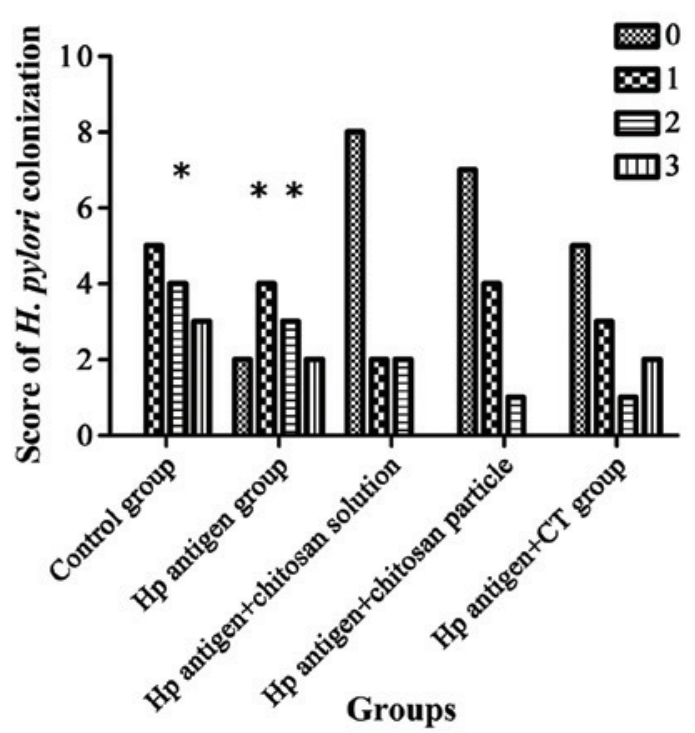

$\mathbf{E}$

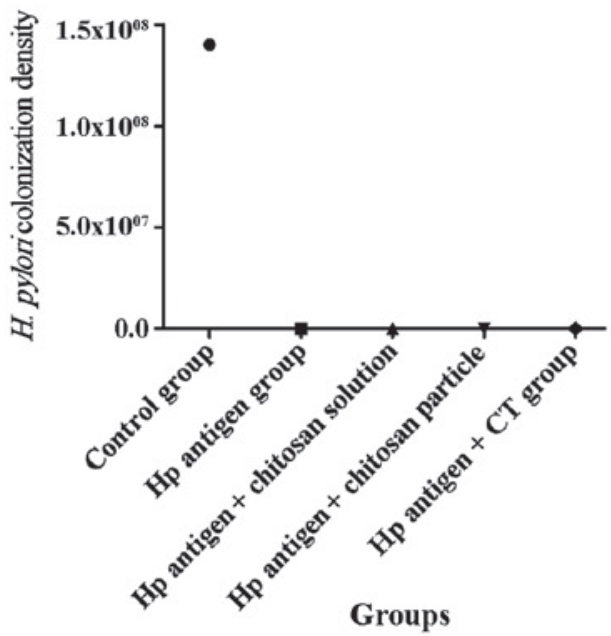

Figure 2. (A) Hp infection in the gastric mucosa after therapeutic vaccination. ${ }^{*} \mathrm{P}<0.005$ compared with the Hp antigen + chitosan solution group and the Hp antigen + chitosan particle group; $\mathrm{P}<0.05$ for the control group vs. the Hp antigen + CT group. ${ }^{* *} \mathrm{P}<0.05$ compared with the Hp antigen + chitosan solution group and the Hp antigen + chitosan particle group. (B) The Hp colonization density in the groups with the adjuvants was significantly less when compared with the control group $(\mathrm{P}<0.05)$, and the Hp colonization density in the group with chitosan as the adjuvant was significantly less than that of the Hp antigen group without any adjuvant $(\mathrm{P}<0.05)$. ${ }^{*} \mathrm{P}<0.001$ compared with the Hp antigen + chitosan solution group and the Hp antigen + chitosan particle group; $\mathrm{P}<0.05$ for the control group vs. the Hp antigen $+\mathrm{CT}$ group. ${ }^{* * *} \mathrm{P}<0.05$ compared with the Hp antigen + chitosan solution group and the Hp antigen + chitosan particle group. (C and D) Giemsa staining of the gastric mucosa (magnification, $\mathrm{x} 400)$. (0, nil; 1, 1-2 cells/crypt; 2, 3-10 cells/crypt; 3 , 11-20 cells/crypt; and 4, >21 cells/crypt). (C) The control group revealed greater Hp colonization in the gastric pits as indicated by the red circle. (D) The group with chitosan as the adjuvant demonstrated no Hp colonization in the gastric pits. (E) The Hp colonization density in the group with chitosan as the adjuvant was significantly less than that of the Hp antigen group without any adjuvants $(\mathrm{P}<0.05)$. Hp, H. pylori; CT, cholera toxin.

H. pylori infection among different groups. To determine $H$. pylori infection in the gastric mucosa following the therapeutic vaccination $H$. pylori clearance was measured, and the $H$. pylori colonization scores and density of the quantitative H. pylori culture were obtained. Pathological tests of the gastric mucosa were also conducted (Fig. 2).

After the therapeutic vaccination, the gastric mucosae of $H$. pylori-infected mice were analyzed for $H$. pylori infection. The $H$. pylori clearance in the groups with chitosan as the adjuvant was identified to be significantly greater than that of the mock-immunized control group $(\mathrm{P}<0.005)$ and the $H$. pylori antigen-immunized group without any adjuvant $(\mathrm{P}<0.05)$; in addition, the $H$. pylori clearance in the group with $\mathrm{CT}$ as the adjuvant was significantly greater than that in the control group (Fig. 2A; $\mathrm{P}<0.05$ ). The H. pylori colonization density in the control group was significantly greater than that in any of the other groups (Fig. $2 \mathrm{~B}$ and Table I; $\mathrm{P}<0.05$ ), and the H.pylori colonization density in the groups with chitosan 


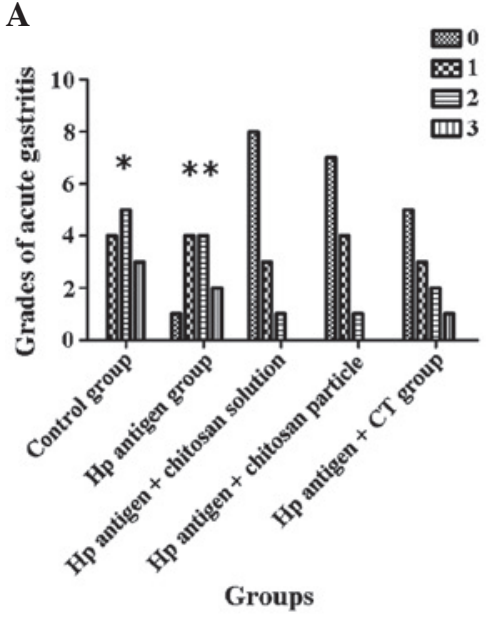

D

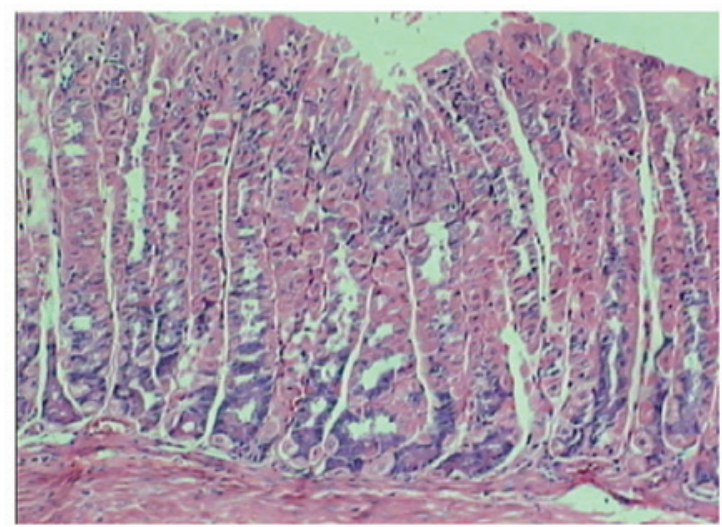

B

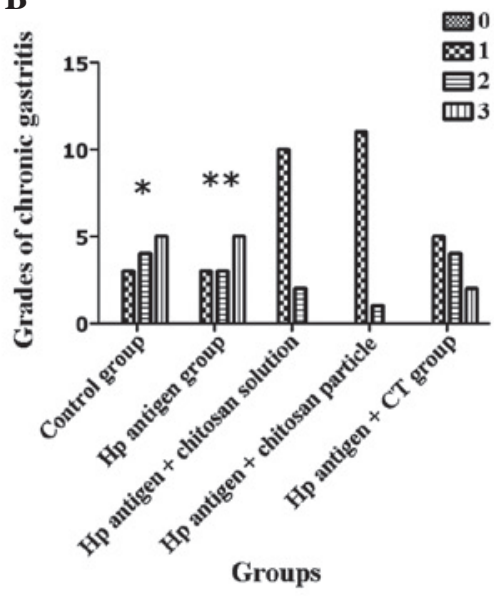

C

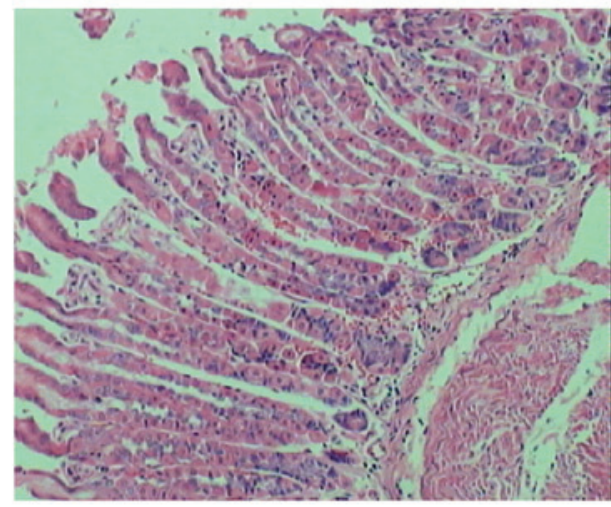

$\mathbf{E}$

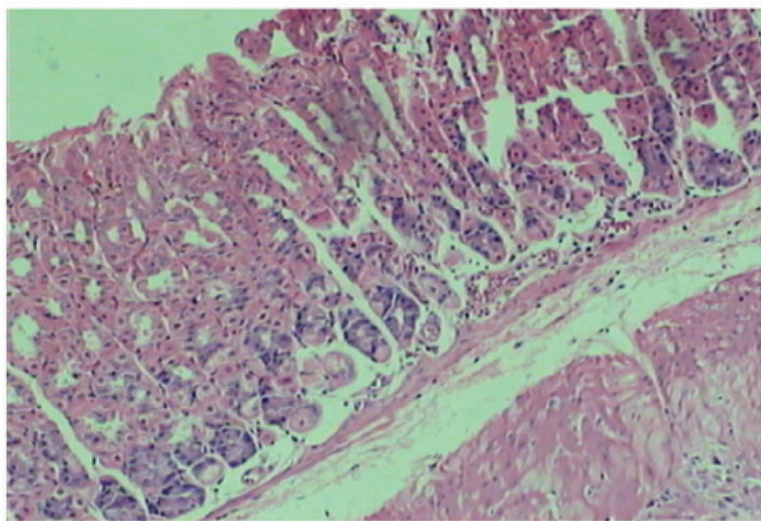

Figure 3. (A) Grade values of acute gastritis. "P<0.001 compared with the Hp antigen + chitosan solution group and the Hp antigen + chitosan particle group; $\mathrm{P}<0.005$ for the control group vs. the Hp antigen $+\mathrm{CT}$ group. ${ }^{* *} \mathrm{P}<0.005$ compared with the Hp antigen + chitosan solution group and the Hp antigen + chitosan particle group; $\mathrm{P}<0.05$ for the Hp antigen group vs. the Hp antigen $+\mathrm{CT}$ group. (B) The grade values of chronic gastritis in the groups with chitosan as the adjuvant were significantly less when compared with those of the groups without chitosan as the adjuvant $(\mathrm{P}<0.001-0.05)$. ${ }^{*} \mathrm{P}<0.001$ compared with the Hp antigen + chitosan solution group and the Hp antigen + chitosan particle group. ${ }^{* *} \mathrm{P}<0.05$ compared with the Hp antigen + chitosan solution group and the Hp antigen + chitosan particle group. (0, few lymphocyte; 1 , sporadic lymphocyte and plasmocyte; 3 , more lymphocyte and plasmocyte; 3 , large number of lymphocyte and plasmocyte). (C-E) H\&E staining of the gastric mucosa (magnification, x100) revealed the following: (C) The control group demonstrated increased inflammatory cell infiltration; (D) the group with chitosan as the adjuvant revealed mild inflammatory cell infiltration; and (E) the group with CT as the adjuvant demonstrated increased inflammatory cell infiltration. Hp, H. pylori; CT, cholera toxin; H\&E, hematoxylin and eosin.

as the adjuvant was significantly less than that of the $H$.pylori antigen group without any adjuvants (Fig. 2E and Table I; $\mathrm{P}<0.05)$. No significant difference was identified between the groups with chitosan as the adjuvant and the group with $\mathrm{CT}$ as the adjuvant (Fig. 2A, B and E; P>0.05).

Grades of gastritis. H. pylori clearance, H. pylori colonization scores, $H$. pylori colonization density of the quantitative culture, and grades of acute and chronic gastritis in the gastric mucosa were measured to determine $H$. pylori infection and gastritis in the gastric mucosa following therapeutic vaccination.

The gastric mucosae of $H$. pylori-infected mice were tested for gastritis after therapeutic vaccination. The grade of acute gastritis in the groups with an adjuvant was identified to be significantly lower than that of the control group and the H.pylori antigen group without any adjuvant (Fig. 3A; $\mathrm{P}<0.05$ ). For acute gastritis after $H$. pylori infection, no difference was observed in the therapeutic effect between the groups with chitosan as the adjuvant and the group with $\mathrm{CT}$ as the adjuvant (Fig. 3A; P>0.05). However, the vaccine with chitosan as the adjuvant relieved chronic gastritis to a greater extent than the vaccine with $\mathrm{CT}$ as the adjuvant (Fig. 3B; $\mathrm{P}<0.05$ ). HE staining of the gastric mucosa of the control group revealed an increased chronic inflammation response (Fig. 3C). The group with chitosan treatment revealed milder chronic inflammation response compared with the group with $\mathrm{CT}$ as adjuvant (Fig. 3D and E).

Levels of $H$. pylori-specific antibodies in the gastric mucosa serum, and saliva. To determine the local immune response induced by the therapeutic vaccination, the levels of H. pylori-specific IgA and IgG antibodies in each group were measured by ELISA.

A significant difference $(\mathrm{P}<0.001)$ was observed between the different groups in the level of anti-H. pylori IgA in the gastric mucosa and saliva, and specific anti-H.pylori $\mathrm{IgG}$ in the sera. The levels of $H$. pylori-specific antibodies in the groups that were treated with a vaccine plus an adjuvant were significantly greater when compared with those of the control group and the H.pylori antigen groups (Fig. 4A and B; $\mathrm{P}<0.01-0.001$ ). In addition, no significant difference was identified between 

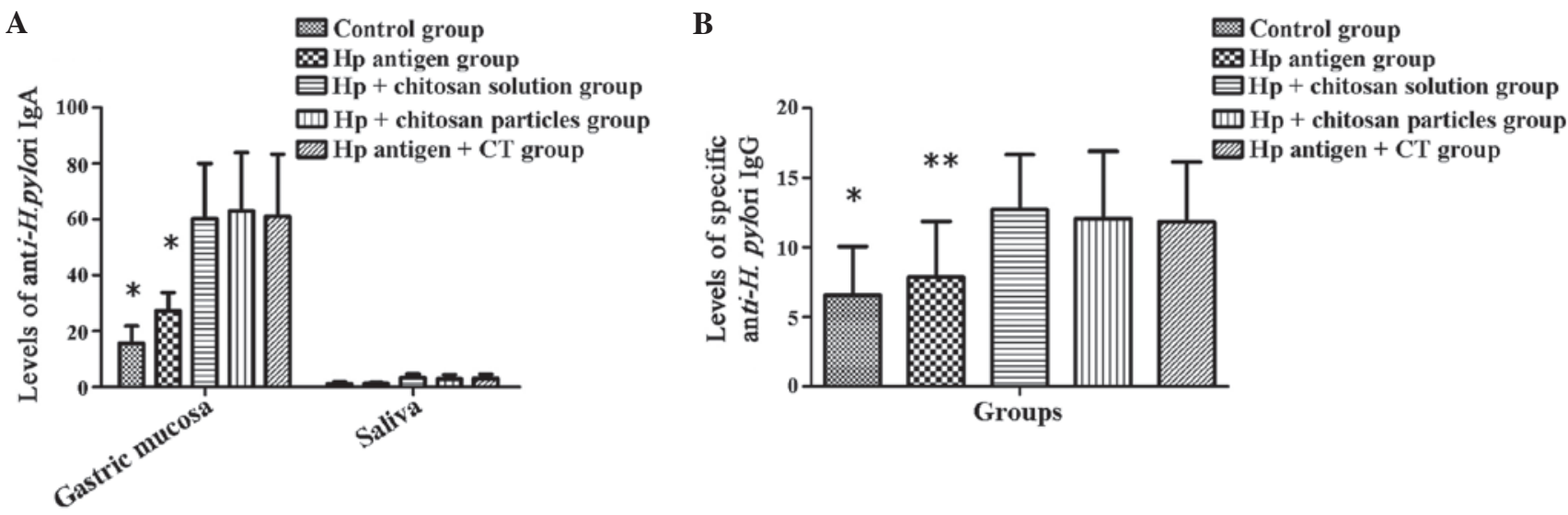

Figure 4. (A) Levels of Hp-specific antibodies in the gastric mucosa and saliva. (B) Levels of Hp-specific antibodies in the sera. ${ }^{*} \mathrm{P}<0.01$ and ${ }^{* *} \mathrm{P}<0.05$, compared with the $\mathrm{Hp}+$ chitosan solution group, the $\mathrm{Hp}+$ chitosan particle group and the Hp antigen + CT group; . Hp, H. pylori; CT, cholera toxin.

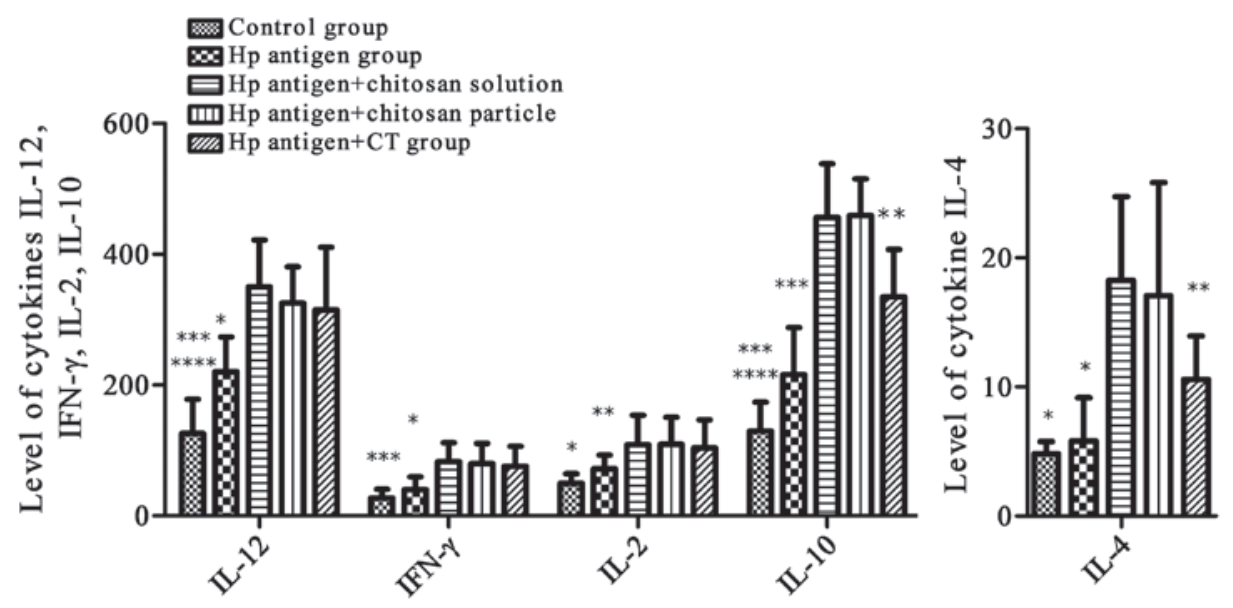

Figure 5. Levels of Hp-specific antibodies in the gastric mucosa, serum and saliva. ${ }^{*} \mathrm{P}<0.01,{ }^{* *} \mathrm{P}<0.05$ and ${ }^{* * *} \mathrm{P}<0.001$ between the Hp antigen group and the $\mathrm{Hp}+$ chitosan solution group, the Hp + chitosan particle group, and the Hp antigen + CT group. Hp, H. pylori; CT, cholera toxin.

the groups with chitosan as the adjuvant and the group with $\mathrm{CT}$ as the adjuvant (Fig. $4 \mathrm{~A}$ and $\mathrm{B} ; \mathrm{P}>0.05$ ).

Effect of TH1 and TH2. To determine the cellular immune response (TH1 and $\mathrm{TH} 2$ ) induced by the therapeutic vaccination, ELISA was conducted to establish the levels of cytokines in the gastric mucosa and the ratio of $\mathrm{IgG} 2 \mathrm{a}$ to $\mathrm{IgG} 1$ in the sera of each group.

The levels of cytokines, IL-12, IFN- $\gamma$, and IL-2 in the groups administered with a vaccine plus an adjuvant were observed to be significantly greater than those of the control group and the H.pylori antigen group $(\mathrm{P}<0.001-0.05)$. The level of IL-10 in the groups administered with a vaccine plus an adjuvant was significantly greater than that of the control and the $H$. pylori antigen groups $(\mathrm{P}<0.001-0.01)$, and the level of IL-10 in the group administered with a vaccine plus chitosan as the adjuvant was identified as significantly greater than that of the group with $\mathrm{CT}$ as the adjuvant $(\mathrm{P}<0.01)$. The level of IL-4 in the group administered with a vaccine plus chitosan as the adjuvant was significantly greater than that of the control group, the $H$. pylori antigen group and the group with $\mathrm{CT}$ as the adjuvant $(\mathrm{P}<0.001-0.05)$. The level of IL-10 in the group administered with a vaccine plus $\mathrm{CT}$ as the adjuvant

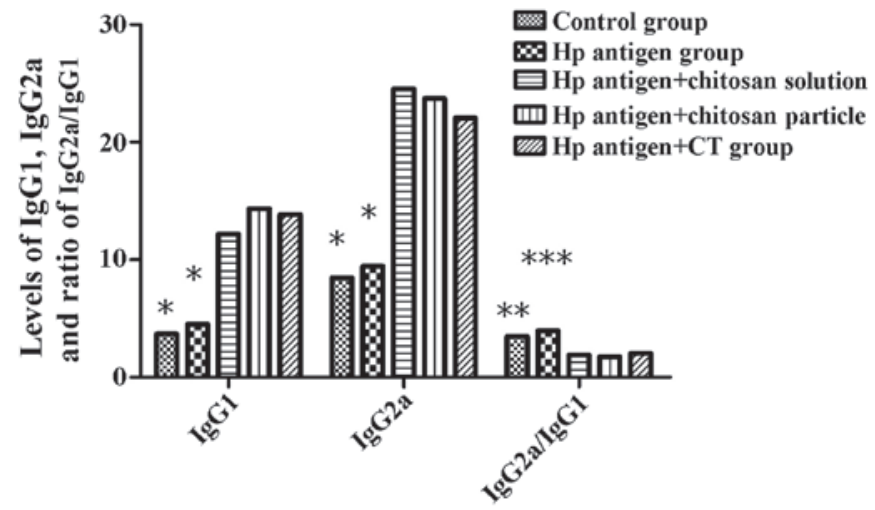

Figure 6. Levels of $\operatorname{IgG} 1$ and $\operatorname{IgG} 2 \mathrm{a}$, and ratio of $\operatorname{IgG} 2 \mathrm{a}$ to $\operatorname{IgG} 1$ in the sera. ${ }^{*} \mathrm{P}<0.001$ vs. the Hp antigen + chitosan solution group, the Hp antigen + chitosan particle group, and the Hp antigen $+\mathrm{CT}$ group. ${ }^{* *} \mathrm{P}<0.05$ vs. the Hp antigen + chitosan solution group and the Hp antigen + chitosan particle group. ${ }^{* * *} \mathrm{P}<0.01$ vs. the $\mathrm{Hp}$ antigen + chitosan particle group, ${ }^{* * *} \mathrm{P}<0.05$ when compared with the Hp antigen + chitosan solution group and the Hp antigen + CT group. Hp, H. pylori; CT, cholera toxin.

was found to be significantly greater than that of the control group (Fig. 5; $\mathrm{P}<0.05$ ). 
A

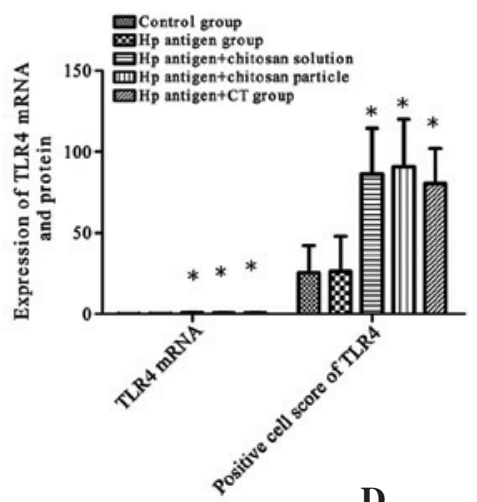

C

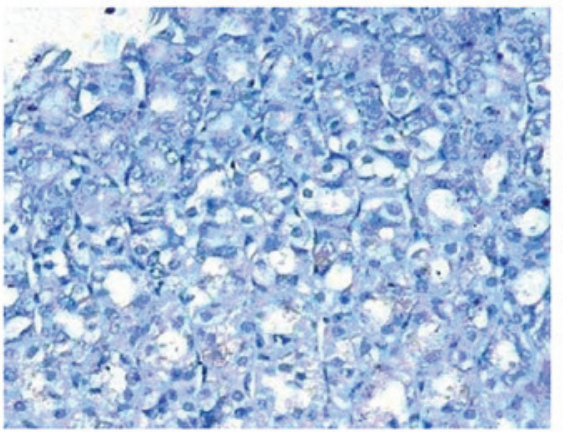

D

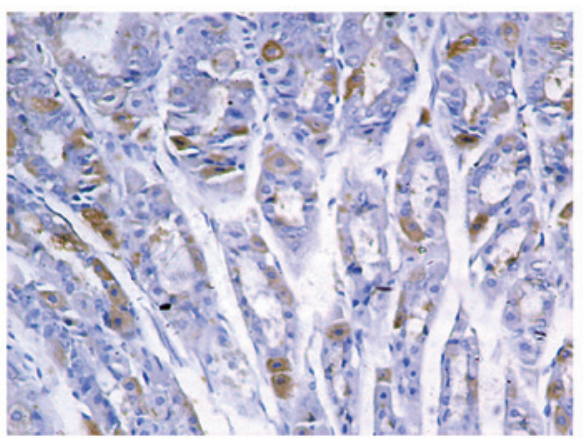

B

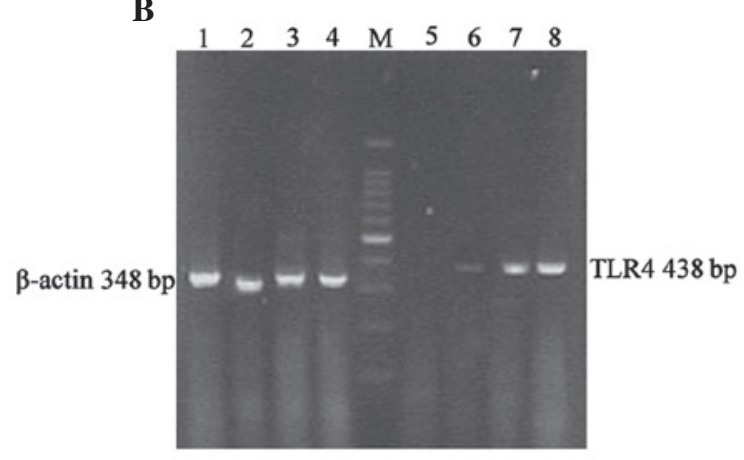

$\mathbf{E}$

Figure 7. TLR4 mRNA and protein expression levels in the gastric mucosa. (A) * $\mathrm{P}<0.001$ compared with the control group and the Hp antigen group. (B) Lane M, 100-bp DNA ladder; lanes 1-4, $\beta$-actin; lanes 5-8, TLR4; lanes 1 and 5, control group; lanes 2 and 6, Hp antigen group; and lanes 3 and 7, Hp antigen + chitosan solution group; lanes 4 and 8, Hp antigen + chitosan particle group. (C-E) TLR4 immunohistochemical staining of the gastric mucosa (magnification, x400). (C) The control group showed few positive cells. (D) The group with chitosan as the adjuvant showed abundant positive cells. (E) The group with CT as the adjuvant showed abundant positive cells. No significant difference was observed between the group with chitosan as the adjuvant and the group with CT as the adjuvant ( $\mathrm{P}>0.05)$. Hp, H. pylori; CT, cholera toxin.
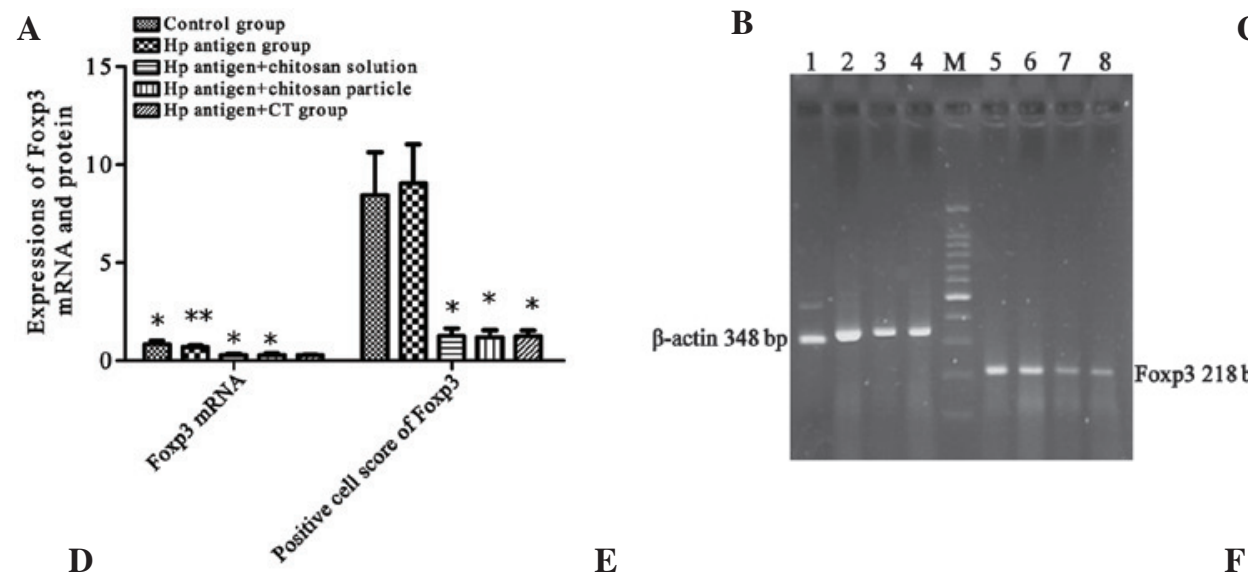

C

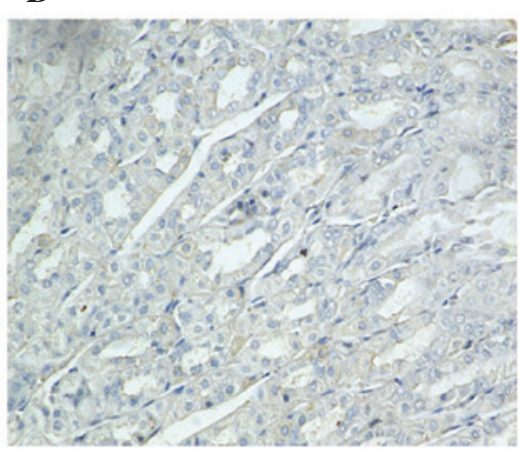

$\mathbf{E}$

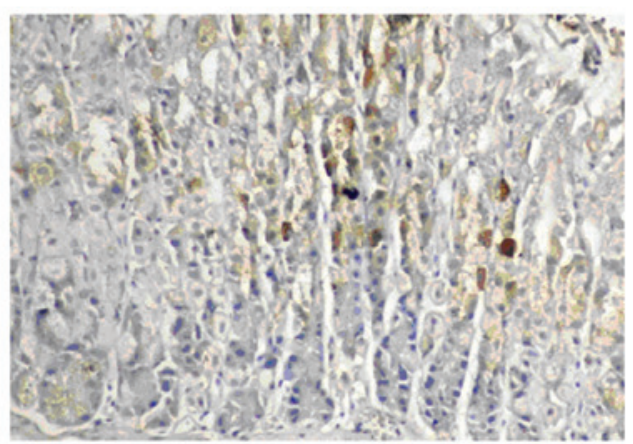

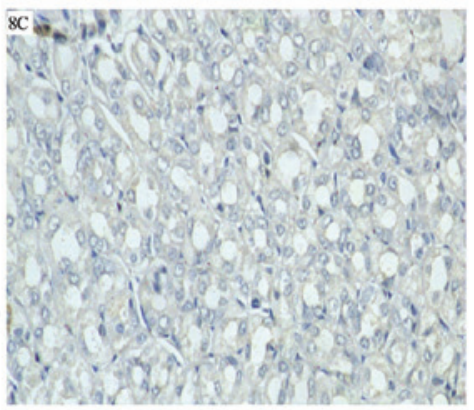

$\mathbf{F}$

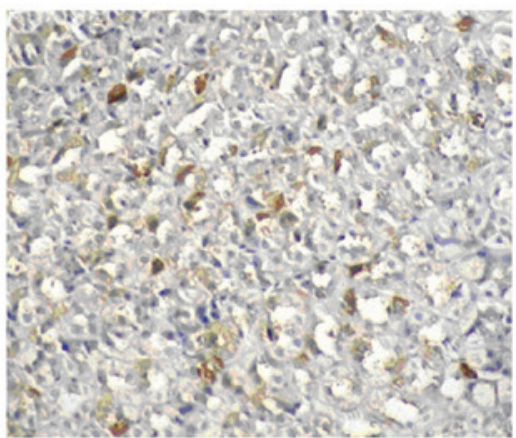

Figure 8. mRNA and protein expression levels of Foxp3 in the gastric mucosa. (A) ${ }^{*} \mathrm{P}<0.001$ vs. the control group and the Hp antigen group; ${ }^{* *} \mathrm{P}<0.05$ vs. the control group. (B) Lane M, 100-bp DNA ladder; lanes 1-4: $\beta$-actin; lanes 5-8, Foxp3; lanes 1 and 5, control group; lanes 2 and 6, Hp antigen group; lanes 3 and 7 , Hp antigen + chitosan solution group; lanes 4 and 8, Hp antigen + chitosan particle group. (C-F) Foxp3 immunohistochemical staining of the gastric mucosa (magnification, x200). (C) The group with chitosan as the adjuvant demonstrated no positive cells. (D) The group with CT as the adjuvant showed no positive cells. (E) The control group revealed sporadic positive cells. (F) The Hp antigen group exhibited abundant positive cells. Hp, H. pylori; CT, cholera toxin. 
Table I. Helicobacter pylori (Hp) colonization density of a quantitative culture in the gastric mucosa.

Median Hp colonization density (colony forming units/g

\begin{tabular}{|c|c|c|}
\hline Group & $\mathrm{n}$ & of gastric mucosa) \\
\hline Control & 12 & $1.4 \times 10^{8}$ \\
\hline Hp antigen & 11 & $1.8 \times 10^{5 \mathrm{a}}$ \\
\hline $\begin{array}{l}\text { Hp antigen }+ \text { chitosan } \\
\text { solution }\end{array}$ & 12 & $0^{\mathrm{ab}}$ \\
\hline $\begin{array}{l}\text { Hp antigen }+ \text { chitosan } \\
\text { particle }\end{array}$ & 12 & $0^{\mathrm{ab}}$ \\
\hline $\begin{array}{l}\text { Hp antigen }+ \text { cholera } \\
\text { toxin }\end{array}$ & 11 & $0^{\mathrm{a}}$ \\
\hline
\end{tabular}

$\mathrm{P}<0.001$. ${ }^{\mathrm{a}} \mathrm{P}<0.001$ compared with the control group; ${ }^{\mathrm{b}} \mathrm{P}<0.05$ compared with the Hp antigen group.

The ratio of $\mathrm{IgG} 2 \mathrm{a}$ to $\mathrm{IgG} 1$ in the sera of the groups administered with a vaccine plus an adjuvant was significantly less than that of the control and the $H$. pylori antigen groups (Fig. 6; $\mathrm{P}<0.01-0.05$ ).

TLR4 $m R N A$ and protein expression levels in the gastric mucosa. To determine the role of TLR4 in vaccination therapy following $H$. pylori infection, TLR4 mRNA and protein expression levels in the gastric mucosa were measured by RT-PCR and immunohistochemical staining. The primer to amplify the 348-bp $\beta$-actin cDNA were as follows: Forward, GAC GAT ATC GCT GCG CTG and reverse, GTA CGA CCA GAG GCA TAC AGG. The primer to amplify the 438-bp TLR4 cDNA were as follows: Forward, CAG CTT CAA TGG TGC CAT CA and reverse, CTG CAA TCA AGA GTG CTG AG.

The expression of TLR4 mRNA and the positive cell scores of TLR4 in the gastric mucosa of the vaccine groups were found to be significantly greater than those of the control and the H. pylori antigen groups (Fig. 7A-E; $\mathrm{P}<0.001)$.

Expression levels of Foxp3 mRNA and protein in the gastric mucosa. To determine the role of Foxp3 in vaccination therapy following $H$. pylori infection, the expression levels of Foxp3 mRNA and protein were measured in the gastric mucosa by RT-PCR and immunohistochemical staining. In RT-PCR, the amplification primers were: Forward, 5'-GAC GAT ATC GCT GCG CTG-3'; and reverse, 5'-GTA CGA CCA GAG GCA TAC AGG-3' for $\beta$-actin (cDNA length, $348 \mathrm{bp}$ ); and forward, 5'-GGC CCT TCT CCA GGA CAG A-3' and reverse, 5'-GCT GAT CAT GGC TGG GTT GT-3' for Foxp3 (cDNA length, 218 bp).

The expression of Foxp3 mRNA and the positive cell score of Foxp3 in the gastric mucosa of the vaccine groups were identified to be significantly less than those of the control and the H. pylori antigen groups (Fig. $8 \mathrm{~A}-\mathrm{F} ; \mathrm{P}<0.001$, vaccine groups, vs. control and $H$. pylori antigen groups).

\section{Discussion}

To investigate the H.pylori therapeutic vaccine with chitosan as the adjuvant, mice were infected with $H$.pylori and vaccinated with an $H$. pylori protein vaccine with chitosan as the adjuvant to delineate the therapeutic effect of the H.pylori vaccine and establish the potential mechanism against $H$.pylori infection in comparison with an $H$. pylori vaccine with $\mathrm{CT}$ as the adjuvant. It was found that the effect of the $H$.pylori therapeutic vaccine with chitosan as the adjuvant was equivalent to the $H$. pylor $i$ therapeutic vaccine with the traditional mucosal adjuvant, $\mathrm{CT}$.

In the present study, the eradication rate of the H. pylori vaccine with chitosan as the adjuvant was found to be $58.33 \%$, which was approximately that of the $H$. pylori vaccine with CT as the adjuvant (45.45\%), and significantly greater $(\mathrm{P}<0.005-0.05)$ than that of the H. pylori antigen alone and control groups. These results indicate that chitosan may act as a substitute for $\mathrm{CT}$ as a mucosal adjuvant for a $H$. pylori therapeutic vaccine. Furthermore, in mice where the $H$. pylori infection had not been eradicated, the density of $H$. pylori colonization in the gastric mucosa of the $H$. pylori-vaccinated groups with chitosan as the adjuvant was significantly $(\mathrm{P}<0.05)$ less than that of the group with $\mathrm{CT}$ as the adjuvant, demonstrating that the adjuvant activity of chitosan was stronger than that of CT. Therefore, the present study demonstrated that the degree of chronic gastritis in the groups with chitosan as the adjuvant was significantly less than that of the groups without chitosan as the adjuvant; thus, indicating that the $H$. pylori vaccine with chitosan as the adjuvant reduced $H$. pylori-induced chronic gastritis.

The effect of humoral immunity in $H$. pylori immunotherapy was also investigated in the present study. It was found that the H.pylori vaccine with chitosan or CT as the adjuvant elicited anti-H. pylori IgG in mice, which may be utilized in immunotherapy. Furthermore, chitosan as the adjuvant in the $H$. pylori vaccine induced a systemic humoral immune response. Similarly, in a study of chitosan co-administered with an influenza virus subunit vaccine, local and serum antibody levels were observed to be markedly enhanced (9). In the present study, it was found that the level of anti-H. pylori IgA in saliva and the gastric mucosa in the groups with chitosan as the adjuvant was significantly greater than that of the control group and the groups without any adjuvant (although it was not significantly different from that of the group with CT as the adjuvant). These results indicate that the local secretory IgA antibody may correlate well with the protection function of the $H$. pylori vaccine. Similarly, IgA has previously been presented as critical in immune protection of an $H$. pylori vaccine with CT as the adjuvant (10) and immune protection of an H.felis vaccine with LT as the adjuvant (11).

The levels of Th1 cytokines (IFN- $\gamma$ and IL-12) and Th2 cytokines (IL-10 and IL-4) in the groups administered with a vaccine plus an adjuvant were significantly greater than those of the control group and the groups without an adjuvant. Furthermore, it was found that the H. pylori vaccine with chitosan or CT as the adjuvant significantly increased the levels of Th1 and Th2 cytokines in the gastric mucosa of mice, as well as in the sera. The increased anti-H. pylori $\operatorname{IgG} 2 \mathrm{a}$ and $\mathrm{IgG1}$ levels may induce the mixed immune response of Th1 and Th2. In addition, the ratio of IgG2a to IgG1 in the 
serum of the groups with chitosan as the adjuvant was found to be less than that of the group with CT as the adjuvant. In addition, the levels of Th2 cytokines, particularly IL-4, in the gastric mucosa of the groups with chitosan as the adjuvant were significantly greater than those of the group with CT as the adjuvant. These results reveal that the $H$. pylori vaccine with chitosan as the adjuvant may recover $\mathrm{Th} 2$ immunity that is suppressed by the $H$.pylori infection, as well as regulate the balance of Th1 and Th2 inflammatory cells, thus facilitating clearance of the H.pylori infection. A previous study reported that IFN- $\gamma$, produced by Th1 cells, induced an increase of the major histocompatibility complex II antigen to recruit inflammatory cells to the gastric mucosa to damage the mucosal tissue, which benefited the clearance of $H$. pylori to a certain degree (12). The H. pylori vaccine with Freund's adjuvant was identified to be more effective against the H.pylori infection by inducing the mixed reaction of Th1 and Th2 rather than only inducing the reaction of Th2 (13). It was demonstrated in the present study that the effective treatment of $H$. pylori infection by the $H$. pylori vaccine was closely associated with Th1 and Th2 cells. Therefore, chitosan as the adjuvant in the $H$. pylori vaccine may be more effective than $\mathrm{CT}$ as the adjuvant for the immunotherapy of $H$. pylori infection, as it is less toxic.

In the present study, following therapeutic vaccination, the expression of TLR4 mRNA in the gastric mucosa and the number of TLR4-positive cells were found to be significantly increased. The low response to $H$. pylori in the gastric epithelial cells may be corrected and the $H$. pylori immune tolerance might be damaged, which may benefit clearance of the $H$. pylori infection. Furthermore in the present study, it was observed that in the vaccination group, the expression of TLR4 mRNA in the gastric mucosae of the mice where the $H$. pylori infection had been eradicated was significantly greater than that of the mice where the H.pylori infection had not been eradicated. This indicates that TLR4 may benefit the antibody-mediated $H$. pylori clearance, which is critical in $H$. pylori vaccine immunotherapy. This result is in accordance with a previous study in which TLR4 was determined to be essential for antibody-mediated clearance of bacteria (14).

The effect of regulatory T cells in $H$. pylori immunotherapy was also investigated in the present study. The changes of Foxp3 mRNA and Foxp3-positive cells in the gastric mucosa following vaccination were analyzed and Foxp3 mRNA and the number of Foxp3-positive cells in the gastric mucosa were observed to be significantly reduced. Vaccination reduced the level of $\mathrm{CD} 4^{+} \mathrm{CD} 25^{+} \mathrm{Foxp} 3^{+}$Treg in the gastric mucosa, relieved the immune response depression and damaged the $H$. pylori immune tolerance, thus benefiting the $H$. pylor $i$ infection clearance. In addition, it was found that the expression of Foxp3 mRNA in the gastric mucosa of mice where the $H$. pylori infection had been eradicated was significantly less than that in the mice where the H. pylori infection had not been eradicated. Previous studies with an $H$. pylori infection mouse model demonstrated that a certain degree of inflammation is required to reduce the bacterial load in the stomach and that the absence of regulatory $\mathrm{T}$ cells is associated with increased gastric inflammation and a decreased bacterial load (15-17), indicating that the enhancement of Treg, induced by $H$. pylori infection, may inhibit the immune response. It was found in the present study that Treg, in CD25-depleted mice, reduced $H$. pylori loads in the $H$. pylori-infected gastric mucosa, increased the numbers of mucosal T cells, B cells and macrophages, and increased the titers of $H$. pylori-specific IgG1 and IgG2a antibodies. These results indicated that the depression of Treg favored H. pylori clearance. Therefore, after $H$. pylori vaccine inoculation, the significant depression of $\mathrm{CD} 4^{+} \mathrm{CD} 25^{+} \mathrm{Foxp} 3^{+}$Treg in the gastric mucosa may participate in the process of $H$. pylori infection clearance.

In conclusion, the $H$. pylori vaccine with chitosan as the adjuvant was found to effectively increase the $H$. pylori elimination rate. Furthermore, the $H$. pylori vaccine with chitosan or $\mathrm{CT}$ as the adjuvant induced specific anti-H.pylori Ig A in the gastric mucosa, and non-specific secretory $\operatorname{Ig} \mathrm{A}$ and specific anti-H. pylori $\operatorname{IgG}$ in the sera. In addition, the $H$. pylori vaccine with chitosan or $\mathrm{CT}$ as the adjuvant promoted Th1 and Th2 cytokines, and decreased the ratio of IgG2a to IgG1. The H. pylori vaccine with chitosan as the adjuvant effectively increased the humoral immune response, the Th1 and Th2 cell immune reaction, as well as balancing the Th1 and Th2 response; all of which may contribute to clearance of the $H$. pylori infection. Furthermore, following $H$. pylori vaccination, the TLR4 expression in the mouse gastric epithelial cells increased and the number of $\mathrm{CD} 4{ }^{+} \mathrm{CD} 25^{+} \mathrm{Foxp}^{+}$Treg decreased, which may influence the process of $H$. pylori infection clearance. The effect of the $H$. pylori therapeutic vaccine with chitosan as the adjuvant was equivalent to the $H$. pylori therapeutic vaccine with the traditional mucosal adjuvant, $\mathrm{CT}$. Thus, these findings may promote the use of chitosan as an adjuvant for the $H$. pylori therapeutic vaccine.

\section{Acknowledgements}

The present study was financially supported by the National Natural Science Foundation of China (grant no. 30460052).

\section{References}

1. Sijun $\mathrm{H}$ and Yong X: Helicobacter pylori vaccine: mucosal adjuvant \& delivery systems. Indian J Med Res 130: 115-124, 2009.

2. Barrette RW, Szczepanek SM, Rood D, et al: Use of inactivated Escherichia coli enterotoxins to enhance respiratory mucosal adjuvanticity during vaccination in swine. Clin Vaccine Immunol 18: 1996-1998, 2011.

3. Synowiecki J and Al-Khateeb NA: Production, properties, and some new applications of chitin and its derivatives. Crit Rev Food Sci Nutr 43: 145-171, 2003

4. van der Lubben IM, Verhoef JC, Borchard G and Junginger HE Chitosan and its derivatives in mucosal drug and vaccine delivery. Eur J Pharm Sci 14: 201-207, 2001.

5. Li X, Min M, Du N, Gu Y, Hode T, Naylor M, Chen D, Nordquist RE and Chen WR: Chitin, chitosan, and glycated chitosan regulate immune responses: the novel adjuvants for cancer vaccine. Clin Dev Immunol 2013: 387023, 2013.

6. Kang ML, Kang SG, Jiang HL, et al: In vivo induction of mucosal immune responses by intranasal administration of chitosan microspheres containing Bordetella bronchiseptica DNT. Eur J Pharm Biopharm 63: 215-220, 2006.

7. Xie Y, Zhou NJ, Gong YF, et al: The immune response induced by $H$. pylori vaccine with chitosan as an adjuvant and its relation to immune protection. World J Gastroenterol 13: 1547-1553, 2007.

8. Gong YF, Xie Y, Zhou NJ, et al: Cellular immunity induced by $H$. pylori vaccine with chitosan as an adjuvant. Xi Bao Yu Fen Zi Mian Yi Xue Za Zhi 23: 595-599, 2007 (In Chinese).

9. Bacon A, Makin J, Sizer PJ, et al: Carbohydrate biopolymers enhance antibody responses to mucosally delivered vaccine antigens. Infect Immun 68: 5764-5770, 2000. 
10. Goto T, Nishizono A, Fujioka T, Ikewaki J, Mifune K and Nasu M: Local secretory immunoglobulin A and postimmunization gastritis correlate with protection against Helicobacter pylori infection after oral vaccination of mice. Infect Immun 67: 2531-2539, 1999.

11. Lee CK, Weltzin R, Thomas WD Jr, et al: Oral immunization with recombinant Helicobacter pylori urease induces secretory IgA antibodies and protects mice from challenge with Helicobacter felis. J Infect Dis 172: 161-172, 1995.

12. Saldinger PF, Porta N, Launois P, et al: Immunization of BALB/c mice with Helicobacter urease B induces a T helper 2 response absent in Helicobacter infection. Gastroenterology 115: 891-897, 1998.

13. Eisenberg JC, Czinn SJ, Garhart CA, et al: Protective efficacy of anti-Helicobacter pylori immunity following systemic immunization of neonatal mice. Infect Immun 71: 1820-1827, 2003.
14. Hori S, Nomura T and Sakaguchi S: Control of regulatory T cell development by the transcription factor Foxp3. Science 299: 1057-1061, 2003

15. Litzinger MT, Fernando R, Curiel TJ, Grosenbach DW, Schlom J and Palena C: IL-2 immunotoxin denileukin diftitox reduces regulatory $\mathrm{T}$ cells and enhances vaccine-mediated $\mathrm{T}$-cell immunity. Blood 110: 3192-3201, 2007.

16. Nair S, Boczkowski D, Fassnacht M, Pisetsky D and Gilboa E: Vaccination against the forkhead family transcription factor Foxp3 enhances tumor immunity. Cancer Res 67: 371-380, 2007.

17. Johnson $\mathrm{BD}$, Jing $\mathrm{W}$ and Orentas $\mathrm{RJ}$ : CD $25^{+}$regulatory $\mathrm{T}$ cell inhibition enhances vaccine-induced immunity to neuroblastoma. J Immunother 30: 203-214, 2007. 